\title{
Erratum: "Microwave Oscillations as an Indicator of the Magnetoplasmadynamic Thruster Operation in a Limiting Regime" [Tech. Phys. Lett. 26 (7), 612 (2000)]
}

The list of the authors should read V. I. Brukhty and K. P. Kirdyashev. 